\title{
Surgeon satisfaction and outcomes of tele-proctoring for robotic gynecologic surgery
}

\author{
Amanda M. Artsen ${ }^{1}\left[\right.$ ] Linda S. Burkett $^{1} \cdot$ Umamaheswar Duvvuri $^{2} \cdot$ Michael Bonidie $^{1}$
}

Received: 21 May 2021 / Accepted: 4 July 2021 / Published online: 16 July 2021

(c) The Author(s), under exclusive licence to Springer-Verlag London Ltd., part of Springer Nature 2021

\begin{abstract}
Surgical proctoring requires increasing resources in growing healthcare systems. In addition, travel has become less safe in the era of COVID-19. This study demonstrates surgeon satisfaction and safety with tele-proctoring in robotic gynecologic surgery. This pilot study assesses surgeon satisfaction and operative outcomes with a novel operative tele-proctoring system with a continuous two-way video-audio feed that allows the off-site surgeon to see the operating room, surgical field, and hands of the robotic surgeon. After thorough system testing, two experienced surgeons underwent tele-proctoring for hospital credentialing, completing 7 total cases. Each completed pre- and post-surveys developed from the Michigan Standard Simulation Experience Scale. Surgical characteristics were compared between tele-proctored cases and 59 historical cases proctored in-person over the last 8 years. Surgeons reported unanimous high satisfaction with tele-proctoring $(5 \pm 0)$. There were no major technologic issues. Five of the tele-proctored cases and 35 of controls were hysterectomies. Mean age was $48.2 \pm 1.4$ years, mean BMI was $29.6 \pm 0.9 \mathrm{~kg} / \mathrm{m}^{2}$, and mean uterine weight was $152 \pm 112.3 \mathrm{~g}$. Two-thirds had prior abdominal surgery $(P>0.1)$. Tele-proctored hysterectomies were $58 \pm 6.5 \mathrm{~min}$ shorter than controls $(P=0.001)$. There were no differences in EBL or complication rates $(P>0.1)$. Tele-proctoring resulted in high surgeon satisfaction rates with no difference in EBL or complications. Tele-mentoring is a natural extension of tele-proctoring that could provide advanced surgical expertise far beyond where we can physically reach.
\end{abstract}

Keywords Tele-proctoring $\cdot$ Tele-mentoring $\cdot$ Surgical innovation $\cdot$ Robotic surgery

\section{Introduction}

Since the inception of formalized postgraduate surgical training by Halstead in 1889 , surgical training has sought to pass on knowledge, skills and techniques, primarily through an apprenticeship model [1]. After completing residency, surgeons continue to encounter new technologies and must constantly adapt their skillset to these new opportunities. For example, laparoscopy revolutionized general

Amanda M. Artsen

artsenam@upmc.edu

1 Division of Urogynecology and Reconstructive Pelvic Surgery, Department of Obstetrics, Gynecology, and Reproductive Sciences, University of Pittsburgh, MageeWomens Research Institute, 204 Craft Ave, Pittsburgh, PA 15213, USA

2 Department of Otolaryngology, Head and Neck Surgery, University of Pittsburgh Medical Center, Eye and Ear Institute, Pittsburgh, PA, USA and gynecologic surgery, allowing patients to recover more quickly with fewer complications [2]. More recently, robotic surgery has allowed surgeons to perform surgeries in a minimally invasive fashion for conditions that previously required open surgery.

As with any new technology, hospitals must ensure that surgeons have adequate experience and skills to safely utilize new technologies. Surgical proctoring by expert surgeons allows hospitals to link credentialing to a standardized quality of surgical care across multiple sites [3]. The Society for Gynecologic Surgeons recommends ongoing quality assurance for robotic surgeons to ensure safe patient care and the American College of Obstetricians and Gynecologists recommends that a surgeon undergo proctoring to ensure competency before being granted privileges for new technology $[4,5]$. In addition, proctoring should allow for assessment of the surgical team, environment, and ability to troubleshoot technical failures. This proctoring should be done by someone who has already proven to be competent in the new procedure or technology [5]. 
Certified expert proctors are a limited resource, and due to the small number in our region, it is the current practice at our institution to send expert robotic surgeons to communities up to $3 \mathrm{~h}$ away to provide surgical proctoring. Health care systems will only continue to grow, with the largest $5 \%$ of hospital systems in the United States having 18 or more hospitals. In addition, $43 \%$ of hospitals in the United States are part of one of these large hospital systems [6]. Finally, with the risk of COVID-19 varying dramatically by region, travel has become less safe and in some cases, inadvisable. With these increasing burdens on proctors, a more cost- and time-effective solution that minimizes travel is needed. Teleproctoring uses telemedicine technology to provide surgical oversight from a remote expert surgeon to an on-site surgeon [7]. Studies looking at tele-proctoring in other fields have found this to be feasible, although widespread acceptance is lacking [3, 8-10]. A 2020 literature review found no studies focused on the use of robotic surgery for tele-mentoring or tele-proctoring in gynecology, thus this represents a critical literature gap [11].

We aim to determine feasibility and surgeon satisfaction with novel tele-proctoring services in gynecologic surgery and compare operative outcomes from tele-proctored cases to historical controls, with the ultimate goal of expanding outreach and reducing the burden on expert robotic surgeons.

\section{Methods}

This is a pilot quality-improvement project to develop and implement tele-proctoring of robotic gynecologic surgery. Approval was obtained from the Quality Improvement Review Committee and thus this study does not require additional Institutional Review Board oversight.

Using principles of telemedicine technology and available componentry, we developed a proprietary system for teleproctoring. A camera is mounted in the operative room that allows the proctor to move the camera and pan the operative room. This affords the expert surgeon a view of the operating room table and the hands of the surgeon at the robotic console (Fig. 1). The surgeon's view also includes input from the robotic camera, allowing a clear picture of the surgical field. The video feed is continuous, two-way and includes audio feed for optimal communication. Similar telecommunication systems can be constructed using previously described technology [12]. The total cost was approximately $\$ 14,000$, covered by our hospital as an investment in remote proctoring. Initial testing consisted of a trainee suturing on a DaVinci robot simulator (Intuitive Surgical, Sunnyvale CA) while an expert surgeon provided feedback from a remote location. Testing was then performed in a cadaver lab, where gynecology residents and fellows performed laparoscopic entry, robotic docking, and robotic hysterectomy under

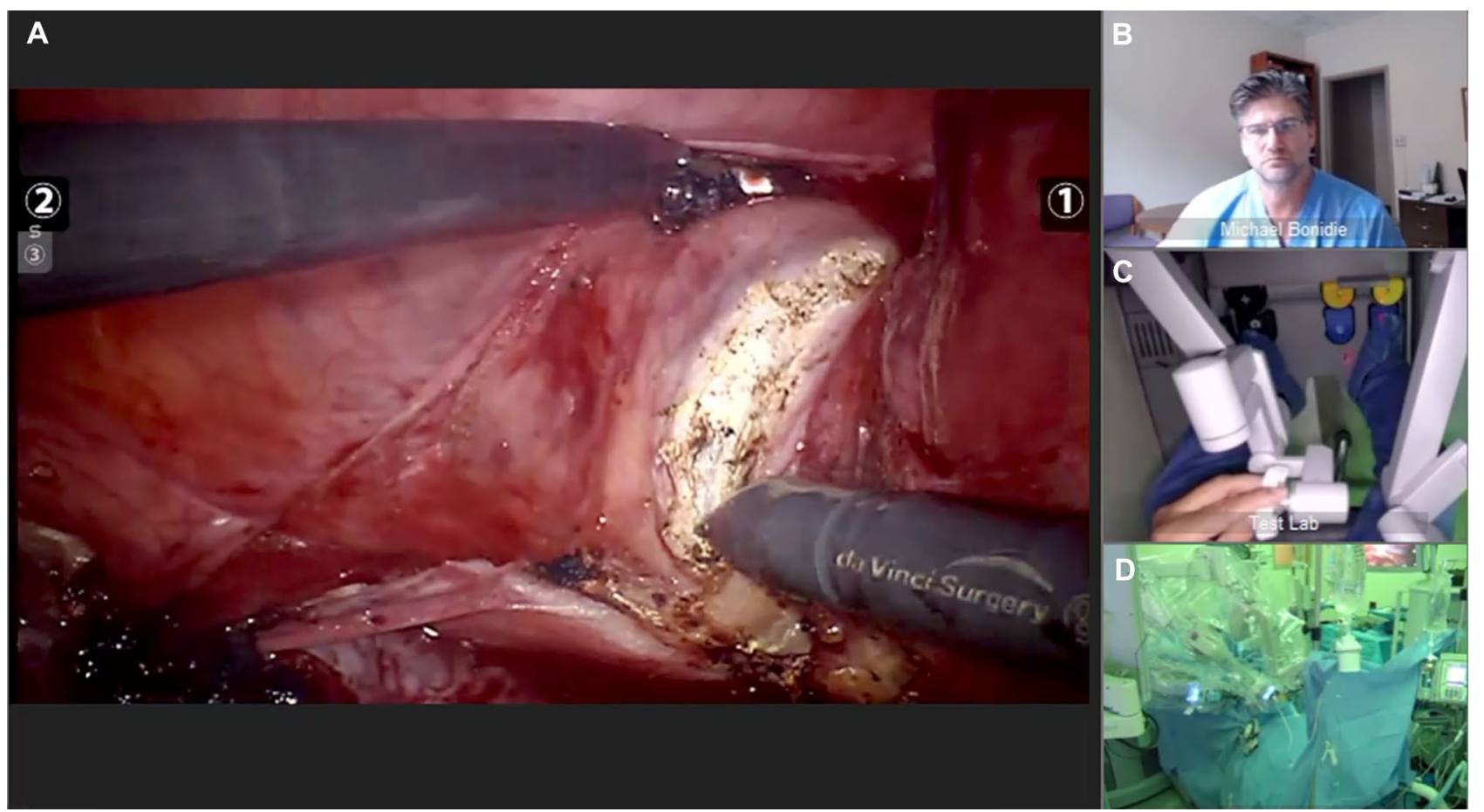

Fig. 1 Tele-proctoring views seen by a proctoring surgeon and operating room team. Tele-proctoring system allows the proctoring surgeon and surgical team in the room to see the robotic camera view $(\mathbf{A})$, the proctor $(\mathbf{B})$, the surgeon's hands at the console $(\mathbf{C})$ and the operating room $(\mathbf{D})$ 
"tele-guidance." Finally, the tele-proctoring system was utilized to proctor a surgeon undergoing formalized surgical proctoring during a robotic hysterectomy on a live person. Here, the expert robotic surgeon utilized tele-proctoring but was available on-site for concerns or system failure. In the event of system failure during live surgery, the proctored surgeon could complete the case in a laparoscopic fashion.

After optimizing the technology in a simulator, cadaver lab and onsite testing, surgeons requesting robotic privileges were invited to participate in tele-proctoring rather than inperson proctoring. Each surgeon underwent one in-person proctoring session prior to initiating tele-proctoring. Each surgeon then underwent 3-5 proctoring sessions depending on their skill level before being credentialed. Pre- and post-surveys were developed from the validated Michigan Standard Simulation Experience Scale (MiSSES) to evaluate surgeon confidence in the procedure and satisfaction with the proctoring process. The MiSSES scale uses a 5-point Likert scale with $5=$ strongly agree, $4=$ agree, $3=$ neither agree nor disagree, $2=$ disagree, $1=$ strongly disagree [13].

Global Evaluative Assessment of Robotic Skills (GEARS) scores were assigned by the proctoring surgeon. The GEARS scale is a 5-point scale which rates surgeons on depth perception, bimanual dexterity, efficiency, force sensitivity, autonomy and robotic control. A low score of 1 in each category represents poor surgical technique such as "unable to complete entire task" or "poor coordination" and a high score of 5 represents mastery, including "expertly uses both hands in a complementary way," and "confident, efficient and safe conduct"[14].

Historical controls consisted of all cases proctored inperson by the same surgeon over the last 8 years. Patient demographics, medical and surgical history, and case characteristics were collected for both tele-proctoring cases and historical controls. All data were deidentified and entered into a secure database (REDCap ${ }^{\circledR}$ 9.7.8, Vanderbilt University).

\section{Statistics}

Demographics, clinical characteristics, and MISSeS and GEARS scores are presented as averages with standard deviations. $T$ tests were used for continuous variables between tele-proctored cases to historical controls and Fisher's Exact tests were used for categorical variables. Statistical analysis was performed at the 0.05 significance level with STATA software (version 15.1; StataCorp, College Station, Texas).

\section{Results}

The 2 surgeon subjects in the study successfully completed robotic credentialing after tele-proctoring. The surgeons were advanced laparoscopists having had completed between 5 and 30 robotic procedures this year and have extensive prior laparoscopic experience, reporting 50-100 laparoscopic procedures per year prior to tele-proctoring. After tele-proctoring, the surgeons reported increased confidence in the procedure $(4.3 \pm 0.8)$, satisfaction with accessibility of the proctor $(4.6 \pm 0.5)$, and unanimous high satisfaction with proctoring $(5 \pm 0$; Fig. 2$)$. Although one surgeon initially reported a preference for in-person proctoring, this preference was reversed after the first tele-proctoring session. There were no safety concerns reported by the surgeons during tele-proctoring, and the proctor was successfully able to provide feedback and assess safety during the cases. There were no major technologic issues including any interruption in the stream, loss of video or audio, poor resolution, or significant lag time. At the completion of their tele-proctoring,
Fig. 2 Proctored surgeon perception of Tele-proctoring. Surgeons demonstrated high levels of confidence in the procedure after proctoring, satisfaction with accessibility of the proctor, and satisfaction with proctoring

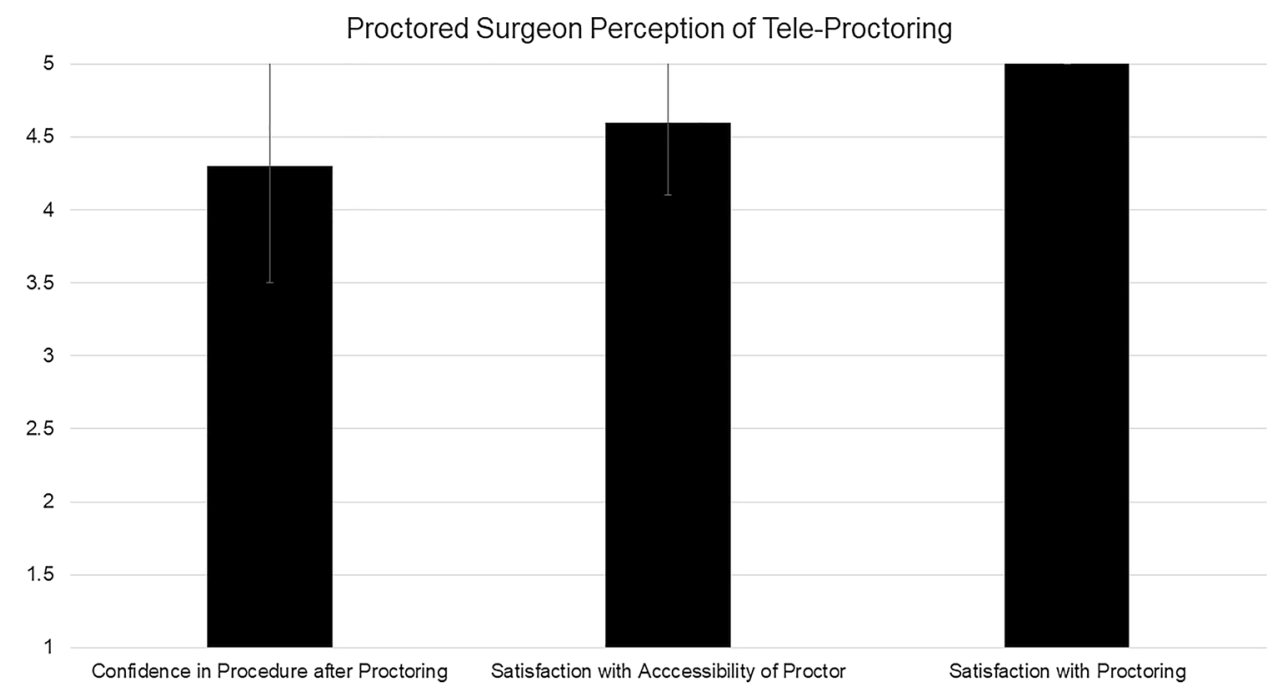


both surgeons demonstrated excellent robotic skills with a GEARS assessment average of 4.3/5.

In comparing tele-proctored cases with historical controls, five of the tele-proctored cases and 35 of controls were total hysterectomies (Table 1). Mean age was $48.2 \pm 1.4$ years, mean BMI was $29.6 \pm 0.9$, and mean uterine weight was $152 \pm 112.3$ with no differences between the groups $(P>0.1)$. Two-thirds had prior abdominal surgery with no differences between the groups $(P=0.52)$. Teleproctored hysterectomies $(n=5)$ were $58 \pm 6.5 \mathrm{~min}$ shorter than in-person cases $(n=35, P=0.001)$. There was no difference in estimated blood loss for hysterectomies. One complication was noted for the tele-proctored cases, requiring a return to the OR for a vaginal cuff separation 1 month postoperatively. Postoperative complications in the historical cohort included 1 conversion to open surgery, 1 case of re-excision of granulation tissue 1 year postoperatively, 3 cases of surgical site infection, 1 case of urinary retention, and 1 urinary tract infection. These complication rates were similar between groups $(P>0.9)$.

\section{Discussion}

We provide one of the first studies to demonstrate the feasibility and surgeon satisfaction of robotic tele-proctoring in gynecology. Proctoring surgeons for credentialing in new technology is necessary for hospitals to ensure delivery of high-quality care but is time-intensive and expensive. Our surgeons routinely drive up to $3 \mathrm{~h}$ away to provide this proctoring, resulting in lost productivity of expert surgeons. the high surgeon satisfaction of tele-proctoring using a system that allows assessment of the surgeon's robotic surgical skill and team functioning. There were no differences in estimated blood loss or rates of surgical complications. Tele-proctored cases were shorter, but this is likely due to variation in surgeon skills and experience and the presence of trainees. All cases were performed within an academic center. Trainees were not present for tele-proctoring cases but may have participated in trocar placement, uterine manipulation and docking for historical cases. We anticipate that using this system would also provide significant cost savings as equipment used for telemedicine can often be repurposed for teleproctoring and would avoid the lost productivity and cost of expert surgeon travel [12]. Even when only accounting for travel costs, it is estimated that $\$ 2446$ was saved over 7 teleproctoring sessions. It is expected, therefore, that the cost of the telementoring equipment would be equaled within 6 years.

Limitations of the study include the small sample size of tele-proctored cases with 2 surgeons. Surgeons included in this pilot study are also high-volume surgeons with excellent laparoscopic experience, an ideal population to pilot this technology in. However, more extensive backup planning may be necessary for surgeons with more limited skills. We currently envision this technology being used to proctor surgeons in new modalities, rather than new procedures, allowing surgeons to fall back on other modalities (laparoscopic, open) in which they are already credentialed if needed. Robotic tele-proctoring may also be appropriate for new graduates after in-person proctoring in these other modalities. In addition, although these cases were all using the $\mathrm{Da}$
Table 1 Characteristics of tele-proctored versus in-person proctored cases

\begin{tabular}{lllc}
\hline & Tele-proctored $(n=7)$ & In-person $(n=59)$ & $P$ value \\
\hline Age $($ years $)$ & $41.9 \pm 13.3^{\mathrm{a}}$ & $48.9 \pm 11.2$ & 0.12 \\
BMI $\left(\mathrm{kg} / \mathrm{m}^{2}\right)$ & $31.0 \pm 11.7$ & $29.4 \pm 6.8$ & 0.73 \\
Prior abdominal surgery & $4(57 \%)$ & $41(70 \%)$ & 0.52 \\
Surgery & & & \\
Total hysterectomy & $5(71.4 \%)$ & $35(59.3 \%)$ & 0.77 \\
Adnexal surgery only & $2(28.6 \%)$ & $5(8.5 \%)$ & 0.21 \\
Sacrocolpopexy & 0 & $16(27.1 \%)$ & 0.33 \\
Myomectomy & 0 & $2(3.4 \%)$ & 0.80 \\
Excision endometriosis & 0 & $1(1.7 \%)$ & 0.90 \\
Uterine weight (grams) & $145.2 \pm 124.4$ & $153.6 \pm 112.4$ & 0.88 \\
Estimated blood loss (mL) & & & 0.13 \\
All cases & $27.1 \pm 17.0$ & $46.5 \pm 32.9$ & 0.33 \\
Hysterectomy only & $34.0 \pm 15.2$ & $50.1 \pm 35.6$ & \\
Operating time (min) & & & $\mathbf{0 . 0 0 0 1}$ \\
All cases & $110.3 \pm 26.8$ & $187.5 \pm 70.6$ & $\mathbf{0 . 0 0 1}$ \\
Hysterectomy only & $124.2 \pm 14.6$ & $182.2 \pm 68.2$ & \\
\hline
\end{tabular}

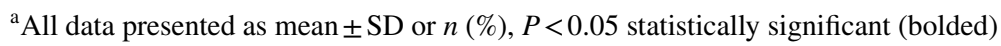

${ }^{\mathrm{b}}$ Myomectomies were excluded in EBL analysis as they are clinically different and numerical outliers 
Vinci robotic system, we have now also demonstrated success with a second robotic system, the Senhance (TransEnterix, Morrisville, NC), increasing the applicability of teleproctoring across multiple robotic technologies.

Tele-proctoring generally is performed merely for credentialing purposes, with the proctor having no legal responsibility to intervene in the setting of a potential complication $[3,15]$. However, tele-mentoring allows the expert surgeon to guide the mentored surgeon to develop new or further develop current skills. Tele-mentoring is a natural extension of the tele-proctoring system that we have developed, and limited studies on tele-mentoring in laparoscopic and robotic surgery in other fields have demonstrated safety and feasibility [16-18]. In our experience, the amount of mentoring needed for each person is very individualized and can range from one to a more extensive mentoring experience over many cases.

A minority of the currently practicing surgeons had the opportunity to learn robotic surgical skills during residency and must now learn these skills by a combination of surgical simulation, which includes the use of models, simulated surgical environments and cadavers, and individual surgical mentoring by an experienced robotic surgeon [4]. As tele-surgery technology develops, tele-mentoring would allow the extension of expertise from large centers to outlying communities while minimizing travel for expert surgeons. This may be especially important now when limiting travel and in-person contact may help decrease the risk of COVID-19 [16, 19]. Current limitations of tele-mentoring include the potential need for a baseline skills assessment to demonstrate the ability of the surgeon to complete the case (perhaps in another modality such as laparoscopic) in the setting of system failure and the inability to physically demonstrate a technique, requiring excellent verbal communication and instruction. New developments such as telestration and remote physical guidance using the movement of a laparoscopic camera or the operation of robotic arms would make tele-mentoring even more feasible [16, 20]. There is technologically no limit to the physical range between the surgeon and the telementor, allowing resources to be available to high need and underserved areas, including international and military applications [21, 22].

\section{Conclusion}

Tele-proctoring is a feasible and safe way to provide proctoring in a large healthcare system while minimizing travel, was associated with high satisfaction in both surgeon and proctor, and may have advantages in cost over traditional in-person proctoring. Tele-proctored cases had similar EBL and complication rates to in-person proctored cases. Telementoring is a natural extension of tele-proctoring and could allow us to extend advanced surgical expertise far beyond where we can physically reach.

Acknowledgements The authors thank the participating surgeons and Max Fedor and Nehal Bhojak from Pittsburgh CREATES for providing technology and logistical support.

Author's contribution A: conceptualization, data collection, manuscript writing. B: conceptualization, results interpretation, manuscript writing. D: conceptualization and actualization. B: conceptualization, data collection, results interpretation, manuscript editing.

Funding This work was supported by NIH/ORWH Building Interdisciplinary Research Careers in Women's Health (BIRCWH) NIH K12HD043441, scholar funds to AMA.

Data availability Original data available by contacting A Artsen.

\section{Declarations}

Conflict of interest The authors declare no conflict of interest. Dr. Duvvuri reports a consulting relationship with Medtronic, unrelated to the current data.

Ethical approval Approval was obtained from the Quality Improvement Review Committee and thus this study does not require additional Institutional Review Board oversight.

Informed consent Informed consent was obtained from surgeons in the study.

\section{References}

1. Rutkow I (2013) The education, training, and specialization of surgeons: turn-of-the-century America and its postgraduate medical schools. Ann Surg 258:1130-1136. https://doi.org/10.1097/ SLA.0b013e3182a6f6a6

2. (2009) ACOG Committee Opinion No 444: choosing the route of hysterectomy for benign disease. Obstet Gynecol 114:1156-1158. https://doi.org/10.1097/AOG.0b013e3181c33c72

3. Heit M (2014) Surgical proctoring for gynecologic surgery. Obstet Gynecol 123:349-352. https://doi.org/10.1097/AOG.0000000000 000076

4. (2015) Committee opinion no. 628: robotic surgery in gynecology. Obstet Gynecol 125:760-767. https://doi.org/10.1097/01.AOG. 0000461761.47981.07

5. Committee on Patient Safety and Quality Improvement (2016) Committee opinion no. 674: guiding principles for privileging of innovative procedures in gynecologic surgery. Obstet Gynecol 128:e85-e88. https://doi.org/10.1097/AOG.0000000000001646

6. Heeringa J, Jones DJ, Machta RM, Furukawa MF, Miller D, and Rich EC. Quality A for HR and Snapshot of U.S. Health Systems. Compendium of U.S. Health Systems Data Highlight No 1:

7. Rosser JC, Gabriel N, Herman B, Murayama M (2001) Telementoring and teleproctoring. World J Surg 25:1438-1448. https://doi. org/10.1007/s00268-001-0129-x

8. McCullough MC, Kulber L, Sammons P et al (2018) Google glass for remote surgical tele-proctoring in low- and middle-income countries: a feasibility study from Mozambique. Plast Reconstr 
Surg Glob Open 6:e1999. https://doi.org/10.1097/GOX.00000 00000001999

9. Datta N, MacQueen IT, Schroeder AD et al (2015) Wearable technology for global surgical teleproctoring. J Surg Educ 72:12901295. https://doi.org/10.1016/j.jsurg.2015.07.004

10. Gambadauro P, Torrejón R (2013) The "tele" factor in surgery today and tomorrow: implications for surgical training and education. Surg Today 43:115-122. https://doi.org/10.1007/ s00595-012-0267-9

11. Murugesu S, Galazis N, Jones BP et al (2020) Evaluating the use of telemedicine in gynaecological practice: a systematic review. BMJ Open 10:e039457. https://doi.org/10.1136/bmjop en-2020-039457

12. Hung AJ, Chen J, Shah A, Gill IS (2018) Telementoring and telesurgery for minimally invasive procedures. J Urol 199:355-369. https://doi.org/10.1016/j.juro.2017.06.082

13. Seagull FJ, Rooney DM (2014) Filling a void: developing a standard subjective assessment tool for surgical simulation through focused review of current practices. Surgery 156:718-722. https:// doi.org/10.1016/j.surg.2014.04.048

14. Goh AC, Goldfarb DW, Sander JC et al (2012) Global evaluative assessment of robotic skills: validation of a clinical assessment tool to measure robotic surgical skills. J Urol 187:247-252. https://doi.org/10.1016/j.juro.2011.09.032

15. Zorn KC, Gautam G, Shalhav AL et al (2009) Training, credentialing, proctoring and medicolegal risks of robotic urological surgery: recommendations of the society of urologic robotic surgeons. J Urol 182:1126-1132. https://doi.org/10.1016/j.juro.2009. 05.042
16. El-Sabawi B, Magee W (2016) The evolution of surgical telementoring: current applications and future directions. Ann Transl Med 4:391. https://doi.org/10.21037/atm.2016.10.04

17. Sebajang H, Trudeau P, Dougall A et al (2006) The role of telementoring and telerobotic assistance in the provision of laparoscopic colorectal surgery in rural areas. Surg Endosc 20:13891393. https://doi.org/10.1007/s00464-005-0260-0

18. Bilgic E, Turkdogan S, Watanabe Y et al (2017) Effectiveness of telementoring in surgery compared with on-site mentoring: a systematic review. Surg Innov 24:379-385. https://doi.org/10. $1177 / 1553350617708725$

19. Centers for Disease Control and Prevention (2020) Considerations for Travelers-Coronavirus in the US I CDC. In: COVID-19. https://www.cdc.gov/coronavirus/2019-ncov/travelers/travel-inthe-us.html. Accessed 9 June 2020

20. Whitten P, Mair F (2004) Telesurgery versus telemedicine in surgery-an overview. Surg Technol Int 12:68-72

21. Mendez I, Hill R, Clarke D et al (2005) Robotic long-distance telementoring in neurosurgery. Neurosurgery 56:434-440. https:// doi.org/10.1227/01.neu.0000153928.51881.27

22. Ereso AQ, Garcia P, Tseng E et al (2009) Usability of robotic platforms for remote surgical teleproctoring. Telemed J E Health 15:445-453. https://doi.org/10.1089/tmj.2008.0164

Publisher's Note Springer Nature remains neutral with regard to jurisdictional claims in published maps and institutional affiliations. 